\title{
Advances in Bone Tissue Engineering
}

\author{
Chao Le Meng Bao, Erin Y. Teo, Mark S.K. Chong, \\ Yuchun Liu, Mahesh Choolani and Jerry K.Y. Chan
}

Additional information is available at the end of the chapter

http://dx.doi.org/10.5772/55916

\section{Introduction}

\subsection{The need for engineered bone}

While bone is inherently capable of regeneration, complications such as excessive bone loss impede healing, necessitating the use of bone grafts. In the United States alone, an estimated 15 million fractures occurs annually, including 1.6 million hospital admissions for traumatic fractures and 2 million osteoporotic fractures, costing over 60 billion dollars and calling for 1.6 million bone grafts each year [1]; a growing demand for bone grafts is similarly observed worldwide. In such applications, autologous bone grafts continue to be regarded as the "gold standard" for bone repair. However, this may not be practicable in cases involving large bone loss. Additionally, patients suffer from significant donor site morbidity, as well as poor outcomes in older patients [2,3]. Allogeneic bone grafts may alternatively be used, but pose potential risks of immune rejection and pathogen transmission [4]. Additionally, the limited number of donors is unable to cope with the clinical demand. Consequently, alternative approaches to provide efficacious and reliable bone grafts are being actively pursued.

The advent of tissue engineering, where the aim is to generate functional tissue, has raised the possibility of engineering bone in vitro [5]. Over the past few decades, a wealth of progress in bone tissue engineering has been achieved, particularly in cell sources, developing biocompatible and biodegradable scaffolds, designing bioreactors to enhance in vitro osteogenic priming, and identifying growth factors that can induce or promote endogenous bone and vascular formation [6]. Numerous pre-clinical trials with various animal models have produced optimistic results [7].Despite the initial optimism, the lack of translation into a clinical setting suggests significant issues remain, including optimisation of cell sources, choice of biomaterial, in vitro preparation and the mode of delivery. 


\subsection{Normal bone anatomy}

As seen in figure 1, bone tissue is organised into cancellous or cortical bone. Cancellous bone (also referred to as trabecular bone or spongy bone), is porous, providing structural support and organisation for bone marrow interspersed inside. In contrast, cortical bone is the compact bone surrounding the marrow space, and confers mechanical strength to bone. The outer layer that covers the surface of cortical bones is the periosteum, containing mainly blood vessels and osteoblasts, which are activated during appositional growth and bone repair [8].

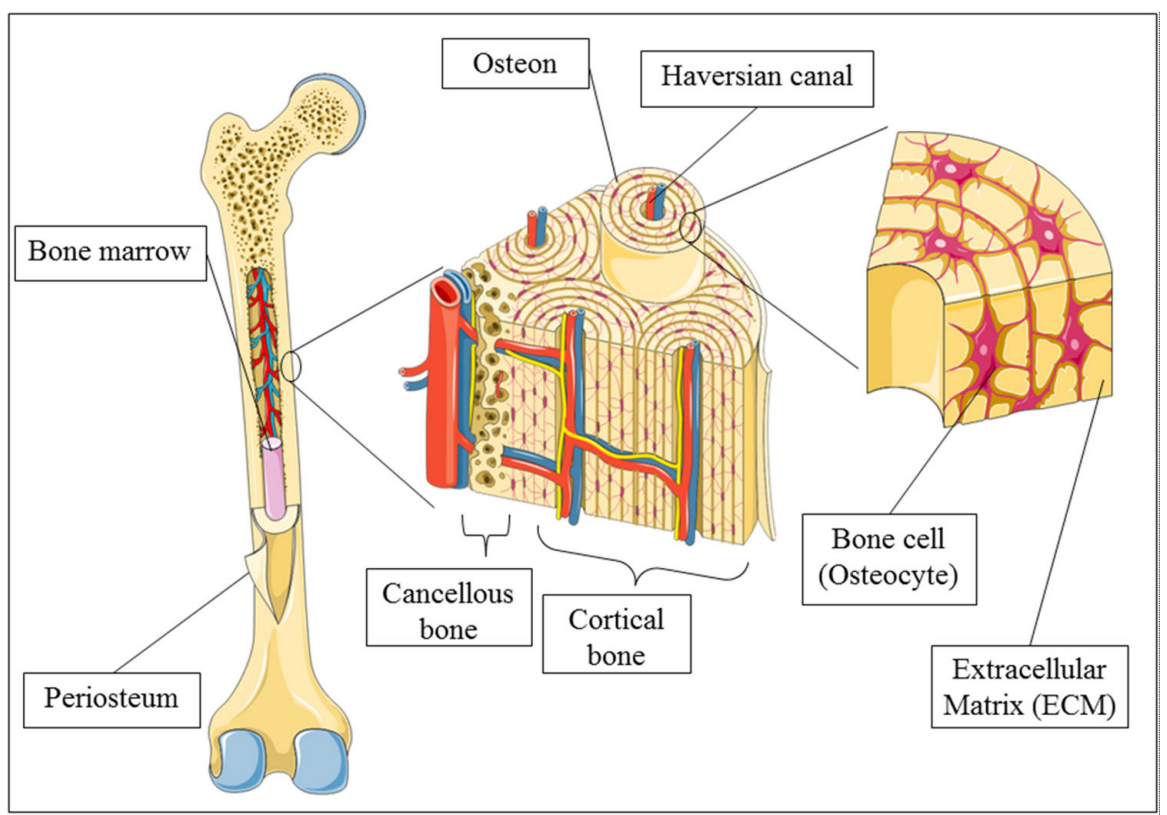

Figure 1. Schematic overview of bone, depicting gross overview, and cellular distribution. (Figures were produced using Servier Medical Art) In particular, osteoprogenitors may be found abundantly in the periosteum and bone marrow, where they perform critical roles in bone repair. Additionally, bone is observed to be highly vascularized, in both the intramedullary canal and periosteal region.

The basic functional unit of the cortical bone is the osteon (or haversian system) which contains cells and extracellular matrix organised in a lamellar pattern, surrounding the haversian canal, in which nerves and blood vessels reside [9]. Within the osteon, osteoblasts and the mature osteocytes exist, contributing to the generation and maintenance of ECM that gives bone it's structural strength [10]. These are derived from osteoprogenitors that reside in the bone marrow and periosteum. Also present are the myeloid-derived osteoclasts that mediate bone resorption; further elaboration on the roles and functions of these cells is provided below. Compared to other tissue, the ECM found in bone is highly mineralized to confer mechanical strength. Calcium and phosphate are found in bone ECM in the form of hydroxyapatite crystal $\left(\mathrm{Ca}_{10}\left(\mathrm{PO}_{4}\right)_{6}(\mathrm{OH})_{2}\right)$, interspersed with Type I collagen [11], while the exact composition remains 
to be elucidated [12]. This unique composite of biological molecules (collagen) and inorganic minerals (calcium phosphate) provides bone with high mechanical strength, as well as toughness to resist impact.

\subsection{Bone physiology}

Bone is a highly dynamic tissue, which undergoes constant remodelling through one's lifetime. Homeostasis is achieved through the combined actions of osteoprogenitors, osteoblasts, osteocytes, as well as osteoclasts. In general, bone formation is effected through the proliferation of osteoprogenitor cells and differentiation into osteoblasts, which are responsible for the regulation of mineralization and collagen production through expression of functional proteins such as osteocalcin and alkaline phosphatase [13,14]. Osteoblasts which get trapped in the ECM eventually differentiate to mature osteocytes forming syncytial networks that function to support bone structure and metabolism. Bone actively undergoes remodelling in response to environmental stimuli, such as physiologic influences or mechanical forces, in accordance with Wolff's law. This process involves a balance between bone formation as described above, as well as bone resorption by osteoclasts [15], and it is crucial for renewing bone, to maintain bone strength and mineral homeostasis [16].

The bone is able to undergo significant regeneration in response to trauma and fractures. Regeneration progresses through the three phases of inflammation, repair and remodelling [17]. The immune system can be seen to be intricately involved in the process of healing and inflammation is required for effective healing [18]. Subsequently, the repair and remodelling processes are initiated in the periosteum, which contain a rich population of osteoprogenitors and osteocytes that proliferate and migrate to the defect site, forming a bony bridge to effect fracture healing. When this bridging does not happen, hypertrophic non-union occurs, necessitating the use of bone grafts.

\section{Stem cell sources}

Over the last few decades, stem cells have emerged as a key player in tissue engineering, both for in vitro generation of bones, and in vivo bone regeneration. Two hypotheses have been proposed to describe the mechanism of healing affected by stem cells. In the conventional approach, osteogenic stem/progenitor cells are proposed to participate in new bone through direct differentiation into functional tissue. More recently, it has emerged that trophic factors secreted by administered stem/progenitor cells can promote functional tissue regeneration [19]. Much research is currently focused on understanding the influence of stem cell origin and culture conditions on clinical outcome.

\subsection{Adult stem cell sources}

The human body retains a small amount of osteoprogenitor cell population with differentiation potential even in adulthood. The main osteogenic progenitor cells - mesenchymal stem cells (MSCs) are considered to consist of a variety of cell populations, with no unique marker to 
delineate a MSC to-date [20]. Therefore, in some studies, the term "mesenchymal stem cells" is used to represent osteoprogenitor cells [21]. The current consensus on the characteristics of a bona-fide MSC should include: 1) expression of cell-surface markers which are non-haemopoietic, non-endothelial and incorporates a number of other cell-surface markers, including CD73, CD105 and CD90, and 2) the capacity for tri-lineage differentiation into osteocytes, chondrocytes and adipocytes [22].Common sources of MSCs include bone marrow, fat tissue and the periosteum [23].

MSCs are most abundantly found in the bone marrow (BM), and BM-MSCs are widely utilised for both autologous or allogeneic transplantations [20]. BM-MSCs can be easily expanded in culture, and induced to differentiate into osteoblasts through culture in a high phosphate environment [24]. In tissue engineering applications, BMMSCs have been used to generate bone graft by loading into appropriate scaffolds, followed by osteogenic culture [25],. BMMSCs may offer favourable characteristics, including availability as an autologous cell source, and thus, non-immunogenic cell source [26]. In contrast to suggestions that BM-MSC are immunoprivilleged and thus suitable for allogeneic - unmatched transplantation [27], a few studies have emerged that reported rejection of murine BM-MSCs following infusion into immune competent recipients [28]. Besides, adult BM-MSCs though advantageous in autologous source availability, have shown relatively low efficiency in osteogenic differentiation in vivo in several comparative studies $[29,30]$.

The periosteum is involved in extraosseous fracture repair, and is thus also thought to contain osteoprogenitors. Indeed, stem cells derived from periosteal tissue showed better mineralization and neovascularization ability than adult bone marrow derived cells and demonstrating good efficacy in healing a calvarial defect model [31]. However, its potential as a cell source is limited by the low volume of periosteum which can be harvested, as well as the complexity of the harvesting process [32]. Further optimization in terms of isolation and culture conditions will be required before it can be a practical source.

Adipose tissue represents another potential source of osteogenic stem cells [33]. Adipose derived stem cells (ADSCs) can be isolated from fat tissues harvested by liposuction or surgical fat section, which are typically considered medical waste [34]. Similar to BM-MSCs, ADSCs respond to osteogenic induction through upregulation of alkaline phosphatase and other key osteogenic proteins, and differentiate into osteoblasts capable of depositing minerals [35]. Additionally, ADSCs have been shown to ne efficacious in bone repair, facilitating the repair of critical size defects in both calvarial and femoral segmental defects [36, 37]. However, the efficacy of bone repair by using ADSCs alone is relatively low compared with marrow-origin MSCs and more primitive MSCs [38, 39].

The adult stem cells represent a rich autologous source for bone tissue engineering, and the longhistory study accumulate mature methodology to facilitate their application in pre-clinical trials or even clinical trial $[40,41]$. Yet the low efficiency of in vitro bone generation and in vivo bone regenerative capacity may call for optimized induction strategy or alternative sources. 


\subsection{Embryonic Stem Cell (ESC) and induced Pluripotent Stem Cell (iPSC)}

In the last decade, the osteogenic induction of embryonic stem cells (ESCs) and the creation of induced pluripotent stem cells (iPSCs) presented new cell sources for bone tissue engineering $[42,43]$. However, expansion of ESCs and iPSCs to clinically useful numbers is logistically challenging, and autologous use is not possible in the case of embryonic stem cells. Moreover, precise control of differentiation is necessary before applying to clinical use. This is especially so since the presence of undifferentiated "rogue cells" may result in tumour formation after transplantation [23].

ESCs are defined by their pluripotency, and can be induced into osteogenic cells through different methods in vitro [44,45]. It is showed that human ESCs can be induced into osteogenic cells and form mineralized bone in vivo without presence of teratoma [46]. However, more investigations will be required to address the potential of teratoma in embryonic stem cells transplantation. Mature methods for specific osteogenic differentiation are also needed to overcome non-specific differentiation before clinical implementation can be contemplated.

As an alternative, iPSCs have recently emerged as a potential cell source for regenerative medicine [43]. Compared to ESCs, iPSCs face fewer ethical challenges, and are able to serve as an autologous cell source. iPSCs have been successfully differentiated into osteoblasts like cells [47], or through induction into a MSC phenotype [48, 49], allowing ready expansion of the iPSC-derived MSC before their direct differentiation into osteoblasts. However, similar issues to that of ESC remain, and concerns remain over the potency of the cells. Additionally, the concept of reprogramming remains poorly understood, and leaving questions on the fate of cells in vivo [49]. Therefore, although the differentiation capacity qualifies the iPSC as a potential source for bone engineering, yet a lot more efforts have to be done before it can be competent.

\subsection{Fetal stem cell sources}

Compared with adult stem cells, fetal stem cells are relatively more primitive, and have higher proliferative and differentiation capacity. MSCs have been derived from various fetal tissues in early to mid-gestation, including the blood, bone marrow, liver, pancreas, kidney thymus and bone [50]. Of these, fetal BM-MSC has shown particular utility for bone tissue engineering as reviewed extensively by Zhang et al [19]. However, its availability may be limited by ethical issues especially where fetal tissues are used to derive MSC [51].

As an alternative source, MSC has also been derived from umbilical cord blood at term gestation, albeit at very low frequencies [51], with optimised protocols achieving up to $60 \%$ success in its derivation [52]. Aside from the cord blood, MSC has also been derived from the umbilical cord vessels and matrix, the placenta and fetal membranes [53]. Although these sources of MSC are plentiful and readily harvested, their utility for bone tissue engineering is somewhat limited compared to fetal bone marrow derived MSC [19, 29]. 


\subsection{Conclusion}

Stem cell sources for bone tissue engineering have been widely explored recently, and several studies have been conducted to compare the different cell sources [21, 29, 30, 44, 54]. Table 1 below summarizes some of these studies and compares the main properties of different stem cell sources.

\begin{tabular}{|c|c|c|c|c|c|}
\hline Cell origin & $\begin{array}{l}\text { Proliferation } \\
\text { rate }\end{array}$ & $\begin{array}{l}\text { Osteogenic } \\
\text { capacity }\end{array}$ & $\begin{array}{c}\text { Mineral } \\
\text { deposition }\end{array}$ & Main Limitations & Key Advantages \\
\hline $\begin{array}{l}\text { Adult bone } \\
\text { marrow }\end{array}$ & Low & Medium & Medium & Limited efficiency & $\begin{array}{c}\text { Autograft } \\
\text { accessibility }\end{array}$ \\
\hline Adipose & Low & Low & Low & Low efficiency & $\begin{array}{c}\text { Autograft } \\
\text { accessibility }\end{array}$ \\
\hline Periosteum & High & High & High & Less resource & $\begin{array}{l}\text { Autograft } \\
\text { accessibility }\end{array}$ \\
\hline $\begin{array}{l}\text { Embryonic } \\
\text { stem cell }\end{array}$ & High & Medium & High & $\begin{array}{l}\text { Non-specific } \\
\text { differentiation }\end{array}$ & $\begin{array}{c}\text { Least mature, } \\
\text { highest potential }\end{array}$ \\
\hline Cord blood & Medium & High & Low & $\begin{array}{c}\text { Autograft } \\
\text { non-accessibility }\end{array}$ & $\begin{array}{l}\text { High potential, } \\
\text { good efficiency }\end{array}$ \\
\hline $\begin{array}{l}\text { Fetal bone } \\
\text { marrow }\end{array}$ & High & Very high & Very high & $\begin{array}{c}\text { Autograft } \\
\text { non-accessibility }\end{array}$ & Highest efficiency \\
\hline
\end{tabular}

Table 1. A comparison of stem cell sources based on comparative studies

\section{Biomaterials}

The development of scaffolds is a major aspect in bone tissue engineering research. On one hand, these scaffolds should be rigid and resilient since they function as the main supporting frame work of bone graft. On the other hand, they should also be porous, biocompatible, osteoinductive and osteoconductive so that bone tissue can regenerate within the scaffolds [55]. In addition, a relatively slow degradation rate is crucial to provide mechanical support prior to complete native bone regeneration. The scaffolds can be made of natural, synthetic materials or suitable composites.

\subsection{Natural materials}

Natural materials applied to bone tissue engineering include biological polymers (such as collagen and hyaluronic acid), as well as inorganic materials (such as hydroxyapatite and tricalcium phosphate). Intuitively, naturally occurring materials in native bone, such as collagen, are favoured as the possess the innate biological cues that favour cell attachment and promote chemotactic response when being implanted in vivo [56]. When used as grafts implanted in vivo, those polymers are readily remodelled by the resident cells to the internal environment. Besides, the fibrous property of polymers allows manipulation during scaffold fabrication, so 
that the scaffold's structure and porosity can be easily controlled [57, 58]. However, the telopeptide within these polymers may be immunogenic, and some of the polymer's nature (poor inherent rigidity and high degradation rate) limit their application in bone repair.

The main minerals in bone matrix, hydroxyapatite (HA) and tri-calcium phosphate (TCP), are other candidates for bone scaffolds. Their mechanical properties are able to provide the mechanical support at the defect area after transplantation. However, these minerals are inherently brittle, and may perform poorly in response to impact. Currently, they are usually combined with polymer materials with higher fracture toughness to achieve optimized performance in bone tissue engineering application [59].

\subsection{Synthetic materials}

As compared to natural materials, synthetic materials may be designed and customised for highly specified chemical and physical properties. These properties contribute to controllable mechanical properties of the scaffolds, including tensile strength, resiliency and degradation rate and to tailor desirable biological outcomes, such as reducing risks of toxicity, immunogenicity and infectionscan [60].Synthetic materials, however, lack bioactive properties such as biocompatibility, osteoinductivity and osteoconductivity, necessitating further modification prior to use.

The most often used synthetic materials for three dimensional scaffolds are saturated poly- $\alpha$ hydroxy esters, including poly lactic acid (PLA), poly glycolic acid (PGA), poly lactic-coglycolic acid (PLGA), and poly caprolactone (PCL) [60]. They can be processed by techniques such as gas forming, phase separation, fused deposition, and 3D printing [61-64]. The choice of polymers and fabrication techniques for three dimensional scaffolds used in tissue engineering are a major aspect in material science, and much progress in this field has been made in the last few decades [65].

As most materials alone showed some form of limitations, now researchers mostly design and fabricate composite materials that combine polymers and inorganic minerals, to let the different nature of materials complement each other, and attain optimal and controllable degradation rate and mechanical properties. The combination can be varies and the fabrication methods are diverse [66].

\section{Emerging research theme: Addressing the need for vascularisation}

\subsection{Vascularisation as limiting bottleneck}

Much effort has been focused on generating tissue engineered bone grafts in vitro, and several attempts have been made to heal bone defects with engineered grafts in vivo. The achievements in bone tissue engineering led considerable progress in finding potent osteogenic cell sources and suitable biomaterials, as well as the development of scaffolds and the use of bioactive factors. Currently, the attention has gradually shifted to strategies to improve vascular 
formation in tissue engineered bones as it emerges as the most crucial factor in ensuring graft survival and hence bone repair.

Having a network of blood vessels within a tissue-engineered graft is important for maintaining cellular survival particularly within the core of large bone grafts [67]. It has been shown that after implantation, neo-bone tissues were found only at where a vascular network was present [68]. Poor angiogenesis has been identified as the main reason for implant failure and is currently acknowledged as a major challenge in tissue engineering [69-71].

Currently, there are various strategies that are under investigation for improving vascularization in tissue engineered grafts. These include the induction of vascularization in vivo, the design of scaffolds specific to improving vascularization, and prevascularization techniques using coculture systems [72]. The prospect of functional vascularized bone graft for defect healing brings a bright future for clinical application.

\subsection{Induction of angiogenesis and vasculogenesis}

Angiogenesis and vasculogenesis are natural vascularization process that occurs in tissue development and wound healing. The endothelial cells function as the main mediator of neovascularization through forming new blood vessels by angiogenesis and they can be expanded by vasculogenesis. In some studies, endothelial cells were used to generate capillary-like structure and connect vessels in vitro [73]. However, it is unclear as to whether these vascular generation approaches are effective in inducing vascularization in engineered bone graft in vivo.

In addition to directly using endothelial cells to form vessel network, some growth factors related to angiogenesis are used as another method to improve vascularization in engineered tissue. These factors include vascular endothelium growth factor (VEGF), platelet-derived growth factor (PDGF) and fibroblast growth factor (FGF) [74]. A major advantage of utilizing growth factors instead of living cells is that the risk of host rejection can be excluded. However, it seems inefficient using growth factors alone, because of the random formation of new vessels within implanted bone [68]. More importantly, the inappropriate delivery of growth factors may induce excessive angiogenesis which could cause severe pathogenic process such as tumour development, atheroscleosis, and proliferative retinopathies $[68,71]$.

\subsection{Scaffold design to promote vascular formation}

Aside from the selection of molecular and cellular mediator for effective vascularization, the choice of material used is also closely related to the vascular formation ability of the engineered bone graft. The scaffolds have been designed to allow vascular in-growth through a macroporous structure and incorporating vascular cues such as with the use of growth factors and/ or cells, rather than just serving as osteoconductive surfaces [75].

Over the past years, the selection of material candidates for bone tissue engineering scaffolds has been focused on the compatibility of bone cell attachment and growth. But now, much attention has also been switched to homing vascular formation [76]. The materials can impact 
the vascularization outcome of the bone graft from two aspects: 1) supporting the endothelial cells growth and forming vessels; 2) incorporating active molecules that help to introduce vascular formation. For example, the usage of silk fibroin and polycaprolactone (PCL) as components for 3D porous scaffolds exhibited a good support to endothelial cell growth and consequent vascularization $[69,77]$; while poly lactic-co-glycolic acid (PLGA) and poly lactideco-glycolide (PLG) scaffolds showed the ability to incorporate VEGFs and release them locally so that angiogenesis was improved [78, 79].

Additionally, the structural properties, such as geometry and porosity can also affect the angiogenic ability of the scaffolds. Narayan and Venkatraman reported that the pore size of scaffolds have a profound influence on the growth of endothelial cells, with enhanced cell growth with smaller pore sizes and lower interpose distances [80]. Currently, the influence of scaffold design on its osteoconductivity and vascular conductivity is still unclear and deserves more investigations [81].

\subsection{The use of stem cells for neo-vascularisation}

Compared to mature endothelial cells, stem/progenitor cell candidates has been shown to exhibit higher proliferative capacity and differentiation potential [82]. Endothelial progenitor cells undergo vasculogenesis and have been shown to improve vascularization through the release of a milieu of angiogenic factors $[83,84]$.

In addition to using endothelial progenitor cells to induce vascularization, stem cell candidates are also needed for bone formation. Therefore, a co-culture system of the cells from different lineages has been proposed by various groups which reviewed comprehensively by Liu et all [72]. Several attempts have been made in generating vascularized bone graft for defect healing through co-culture systems, combine endothelial lineages with osteogenic cell types on different types of scaffolds [85-89].We have recently shown that co-cultured human fetal mesenchymal stem cells (hfMSC) and umbilical cord blood derived endothelial progenitor cells (UCB-EPC) seeded on PCL-TCP macroporous scaffolds induced more neo-vascularization and better bone formation, compared with the use of hfMSC alone [88].

\section{Summary}

A large number of bone grafts are required annually for clinical treatment of severe bone fracture. The limitations in autograft and allograft restricted their clinical application. Alternatively, tissue engineering approach may offer a new solution to produce bone grafts for clinical use. Over the last twenty years, tissue engineering of the bone has made remarkable progress, although the problems of translating into clinical application still remain.

Various types of stem cells have been used to form mineralized bone in vitro. In contrast, there were much fewer studies focused on the healing efficacy and its potential side effects. One main barrier is the complicated in vivo environment, which has profound interactions with the implanted cell types. This is especially so for allogeneic cells, where the host immune 
reaction is likely to play a very important role, with the macrophage system currently being under intense investigations $[90,91]$.

The use of biomaterials and development of scaffolds are especially important for engineering bone grafts, because they need to provide mechanical support during bone repair and bioactive aspect for bone formation. In order to obtain optimal mechanical properties, and high biocompatibility, numerous composite materials were designed to acquire integrated properties from the individual components. Recently, more stringent requirements brought forth in the scaffold design for complete bone healing efficacy, such as inducing neo-vascularization during the formation of bone.

The achievements in engineering bone tissue so far are encouraging, while new challenges and opportunities are bringing the perspective of bone tissue engineering to a new height in clinical application. In the near future, tissue engineering approaches will achieve full tissue transplantation and engineered bone graft will be mature enough for bone fracture repair treatment.

\section{Acknowledgements}

JC received salary support from the National Medical Research Council, Singapore (NMRC/CSA/012/2009).

\section{Author details}

Chao Le Meng Bao ${ }^{1}$, Erin Y. Teo ${ }^{1,2}$, Mark S.K. Chong ${ }^{1,3}$, Yuchun Liu ${ }^{1,3}$, Mahesh Choolani ${ }^{1}$ and Jerry K.Y. Chan ${ }^{1,2,4}$

1 Experimental Fetal Medicine Group, Department of Obstetrics and Gynaecology, Yong Loo Lin School of Medicine. National University of Singapore, Singapore

2 Department of Reproductive Medicine, KK Women's and Children's Hospital, Singapore

3 Division of Bioengineering, School of Chemical and Biomedical Engineering, Nanyang Technological University, Singapore

4 Cancer and Stem Cell Biology Program, Duke-NUS Graduate Medical School, Singapore

\section{References}

[1] Keefe, O, \& Mao, R. J. a. n. d J. Bone tissue engineering and regeneration: from discovery to the clinic-an overview. Tissue engineering. Part B, Reviews, (2011). , 389-392. 
[2] Banwart, J. C, Asher, M. A, \& Hassanein, R. S. Iliac crest bone graft harvest donor site morbidity. A statistical evaluation. Spine, (1995). , 1055-1060.

[3] Goulet, J. A, et al. Autogenous iliac crest bone graft- Complications and functional assessment. Clinical Orthopaedics and Related Research, (1997). , 76-81.

[4] Betz, R. R. Limitations of autograft and allograft: New synthetic solutions. Orthopedics, (2002). , S561-S570.

[5] Meinel, L, et al. Bone tissue engineering using human mesenchymal stem cells: effects of scaffold material and medium flow. Annals of biomedical engineering, (2004). , 112-122.

[6] Salgado, A. J, Coutinho, O. P, \& Reis, R. L. Bone Tissue Engineering: State of the Art and Future Trends. Macromolecular bioscience, (2004). , 743-765.

[7] Cancedda, R, Giannoni, P, \& Mastrogiacomo, M. A tissue engineering approach to bone repair in large animal models and in clinical practice. Biomaterials, (2007). , 4240-4250.

[8] Buck, D. W, \& Dumanian, G. A. Bone Biology and Physiology: Part I. The Fundamentals. Plastic and Reconstructive Surgery, (2012). , 1314-1320.

[9] Ascenzi, M. G, \& Roe, A. K. The osteon: the micromechanical unit of compact bone. Front Biosci, (2012). , 1551-1581.

[10] Klein-nulend, J, Nijweide, P. J, \& Burger, E. H. Osteocyte and bone structure. Curr Osteoporos Rep, (2003). , 5-10.

[11] Aerssens, J, Dequeker, J, \& Mbuyimuamba, J. M. BONE TISSUE COMPOSITION- BIOCHEMICAL ANATOMY OF BONE. Clinical rheumatology, (1994). , 54-62.

[12] Rey, C, et al. Bone mineral: update on chemical composition and structure. Osteoporosis International, (2009). , 1013-1021.

[13] Harada, S, \& Rodan, i. a. n. d G. A. Control of osteoblast function and regulation of bone mass. Nature, (2003). , 349-355.

[14] Szulc, P, et al. Biochemical markers of bone formation reflect endosteal bone loss in elderly men- MINOS study. Bone, (2005). , 13-21.

[15] Clarke, B. Normal bone anatomy and physiology. Clinical journal of the American Society of Nephrology : CJASN, (2008). Suppl 3: , S131-S139.

[16] Cao, X. Targeting osteoclast-osteoblast communication. Nature medicine, (2011). , 1344-1346.

[17] Claes, L, Recknagel, S, \& Ignatius, A. Fracture healing under healthy and inflammatory conditions. Nature reviews. Rheumatology, (2012). , 133-143.

[18] Cottrell, J, \& Connor, J. P. O. Effect of Non-Steroidal Anti-Inflammatory Drugs on Bone Healing. Pharmaceuticals, (2010). , 1668-1693. 
[19] Zhang, Z. Y, et al. The potential of human fetal mesenchymal stem cells for off-the-shelf bone tissue engineering application. Biomaterials, (2012). , 2656-2672.

[20] Mauney, J. R, Volloch, V, \& Kaplan, D. L. Role of adult mesenchymal stem cells in bone tissue engineering applications: current status and future prospects. Tissue engineering, (2005). , 787-802.

[21] Sakaguchi, Y, et al. Comparison of human stem cells derived from various mesenchymal tissues- Superiority of synovium as a cell source. Arthritis and rheumatism, (2005). , 2521-2529.

[22] Dominici, M, et al. Minimal criteria for defining multipotent mesenchymal stromal cells. The International Society for Cellular Therapy position statement. Cytotherapy, (2006). , 315-317.

[23] Robey, P. G. Cell sources for bone regeneration: the good, the bad, and the ugly (but promising). Tissue engineering. Part B, Reviews, (2011). , 423-430.

[24] Prockop, D. J. Marrow stromal cells as stem cells for nonhematopoietic tissues. Science, (1997). , 71-74.

[25] Bianco, P, \& Robey, P. G. Stem cells in tissue engineering. Nature, (2001). , 118-121.

[26] Petite, H, et al. Tissue-engineered bone regeneration. Nature Biotechnology, (2000). , 959-963.

[27] Krampera, M, et al. Bone marrow mesenchymal stem cells inhibit the response of naive and memory antigen-specific T cells to their cognate peptide. Blood, (2003). , 3722-3729.

[28] Eliopoulos, N, et al. Allogeneic marrow stromal cells are immune rejected by MHC class Iand class II-mismatched recipient mice. Blood, (2005). , 4057-4065.

[29] Zhang, Z. Y, et al. Superior osteogenic capacity for bone tissue engineering of fetal compared with perinatal and adult mesenchymal stem cells. Stem Cells, (2009). , 126-137.

[30] Kern, S, et al. Comparative Analysis of Mesenchymal Stem Cells from Bone Marrow, Umbilical Cord Blood, or Adipose Tissue. Stem Cells, (2006). , 1294-1301.

[31] Sakata, Y, et al. Osteogenic potential of cultured human periosteum-derived cells- A pilot study of human cell transplantation into a rat calvarial defect model. Journal of CranioMaxillofacial Surgery, (2006). , 461-465.

[32] Breitbart, A. S, et al. Tissue engineered bone repair of calvarial defects using-cultured periosteal cells. Plastic and Reconstructive Surgery, (1998). , 567-574.

[33] Zuk, P. A, et al. Multilineage cells from human adipose tissue: Implications for cell-based therapies. Tissue engineering, (2001). , 211-228.

[34] Yoshimura, K, et al. Characterization of freshly isolated and cultured cells derived from the fatty and fluid portions of liposuction aspirates. Journal of cellular physiology, (2006). , 64-76. 
[35] Kakudo, N, et al. Bone tissue engineering using human adipose-derived stem cells and honeycomb collagen scaffold. Journal of Biomedical Materials Research Part A, (2008). A(1): , 191-197.

[36] Cowan, C. M, et al. Adipose-derived adult stromal cells heal critical-size mouse calvarial defects. Nat Biotech, (2004). , 560-567.

[37] Peterson, B, et al. Healing of critically sized femoral defects, using genetically modified mesenchymal stem cells from human adipose tissue. Tissue engineering, (2005). , 120-129.

[38] Li, H. W, et al. Bone regeneration by implantation of adipose-derived stromal cells expressing BMP-2. Biochemical and Biophysical Research Communications, (2007). , 836-842.

[39] Niemeyer, $\mathrm{P}$, et al. Comparison of mesenchymal stem cells from bone marrow and adipose tissue for bone regeneration in a critical size defect of the sheep tibia and the influence of platelet-rich plasma. Biomaterials, (2010). , 3572-3579.

[40] Giordano, A, Galderisi, U, \& Marino, I. R. From the laboratory bench to the patients bedside: An update on clinical trials with mesenchymal stem cells. Journal of cellular physiology, (2007). , 27-35.

[41] Henriksson, H. B, et al. Transplantation of human mesenchymal stems cells into intervertebral discs in a xenogeneic porcine model. Spine (Phila Pa 1976), (2009). , 141-148.

[42] Gepstein, L. Derivation and potential applications of human embryonic stem cells. Circulation Research, (2002). , 866-876.

[43] Takahashi, K, et al. Induction of pluripotent stem cells from adult human fibroblasts by defined factors. Cell, (2007). , 861-872.

[44] Shimko, D. A, et al. Comparison of in vitro mineralization by murine embryonic and adult stem cells cultured in an osteogenic medium. Tissue engineering, (2004). , 1386-1398.

[45] Bielby, R. C, et al. In vitro differentiation and in vivo mineralization of osteogenic cells derived from human embryonic stem cells. Tissue engineering, (2004). , 1518-1525.

[46] Kim, S, et al. In vivo bone formation from human embryonic stem cell-derived osteogenic cells in poly(D,L-lactic-co-glycolic acid)/hydroxyapatite composite scaffolds. Biomaterials, (2008). , 1043-1053.

[47] Tashiro, K, et al. Efficient Adipocyte and Osteoblast Differentiation from Mouse Induced Pluripotent Stem Cells by Adenoviral Transduction. Stem Cells, (2009). , 1802-1811.

[48] Villa-diaz, L. G, et al. Derivation of Mesenchymal Stem Cells from Human Induced Pluripotent Stem Cells Cultured on Synthetic Substrates. Stem Cells, (2012). p. N/A-N/A.

[49] Liu, Y, et al. One-Step Derivation of Mesenchymal Stem Cell (MSC)-Like Cells from Human Pluripotent Stem Cells on a Fibrillar Collagen Coating. PLoS One, (2012). , e33225.

[50] Donoghue, O, \& Chan, K. a. n. d J. Human fetal mesenchymal stem cells. Curr Stem Cell Res Ther, (2006). , 371-386. 
[51] Campagnoli, C, et al. Identification of mesenchymal stem/progenitor cells in human firsttrimester fetal blood, liver, and bone marrow. Blood, (2001). , 2396-2402.

[52] Bieback, K, et al. Critical Parameters for the Isolation of Mesenchymal Stem Cells from Umbilical Cord Blood. Stem Cells, (2004). , 625-634.

[53] Shaw, S. W, David, A. L, \& De Coppi, P. Clinical applications of prenatal and postnatal therapy using stem cells retrieved from amniotic fluid. Curr Opin Obstet Gynecol, (2011). , 109-116.

[54] Chen, D. Y, et al. Superior mineralization and neovascularization capacity of adult human metaphyseal periosteum-derived cells for skeletal tissue engineering applications. International Journal of Molecular Medicine, (2011). , 707-713.

[55] Burg, K. J. L, Porter, S, \& Kellam, J. F. Biomaterial developments for bone tissue engineering. Biomaterials, (2000). , 2347-2359.

[56] Stevens, M. M. Biomaterials for bone tissue engineering. Materials Today, (2008). , 18-25.

[57] Glowacki, J, \& Mizuno, S. Collagen scaffolds for tissue engineering. Biopolymers, (2008). , 338-344.

[58] Sundelacruz, S, \& Kaplan, D. L. Stem cell- and scaffold-based tissue engineering approaches to osteochondral regenerative medicine. Seminars in cell \& developmental biology, (2009). , 646-655.

[59] Ramay, H. R. R, \& Zhang, M. Biphasic calcium phosphate nanocomposite porous scaffolds for load-bearing bone tissue engineering. Biomaterials, (2004). , 5171-5180.

[60] Rezwan, K, et al. Biodegradable and bioactive porous polymer/inorganic composite scaffolds for bone tissue engineering. Biomaterials, (2006). , 3413-3431.

[61] Kim, S, et al. Poly(lactide-co-glycolide)/hydroxyapatite composite scaffolds for bone tissue engineering. Biomaterials, (2006). , 1399-1409.

[62] Chen, V. J, \& Ma, P. X. Nano-fibrous poly(l-lactic acid) scaffolds with interconnected spherical macropores. Biomaterials, (2004). , 2065-2073.

[63] Hutmacher, D. W, et al. Mechanical properties and cell cultural response of polycaprolactone scaffolds designed and fabricated via fused deposition modeling. Journal of biomedical materials research, (2001). , 203-216.

[64] Hutmacher, D. W. Scaffolds in tissue engineering bone and cartilage. Biomaterials, (2000). , 2529-2543.

[65] Hutmacher, D. W, et al. State of the art and future directions of scaffold-based bone engineering from a biomaterials perspective. Journal of Tissue Engineering and Regenerative Medicine, (2007). , 245-260. 
[66] Hutmacher, D. W. Scaffold design and fabrication technologies for engineering tissues- state of the art and future perspectives. Journal of Biomaterials Science-Polymer Edition, (2001). , 107-124.

[67] Langer, R. Tissue engineering: perspectives, challenges, and future directions. Tissue engineering, (2007). , 1-2.

[68] Johnson, E. O, Troupis, T, \& Soucacos, P. N. Tissue-engineered vascularized bone grafts: basic science and clinical relevance to trauma and reconstructive microsurgery. Microsurgery, (2011). , 176-182.

[69] Yu, H. Y, et al. Improved tissue-engineered bone regeneration by endothelial cell mediated vascularization. Biomaterials, (2009). , 508-517.

[70] Koike, N, et al. Creation of long-lasting blood vessels. Nature, (2004). , 138-139.

[71] Santos, M. I, \& Reis, R. L. Vascularization in Bone Tissue Engineering: Physiology, Current Strategies, Major Hurdles and Future Challenges. Macromolecular bioscience, (2010). , 12-27.

[72] Liu, Y, Chan, J. K. Y, \& Teoh, S. -H. Review on Vascularised Bone Tissue Engineering Strategies: Focus on Coculture Systems. Journal of Tissue Engineering and Regenerative Medicine, (2012). In Press.

[73] Niklason, L. E, et al. Functional arteries grown in vitro. Science, (1999). , 489-493.

[74] Ahrendt, G, Chickering, D. E, \& Ranieri, J. P. Angiogenic growth factors: A review for tissue engineering. Tissue engineering, (1998). , 117-130.

[75] Mastrogiacomo, M, et al. Role of scaffold internal structure on in vivo bone formation in macroporous calcium phosphate bioceramics. Biomaterials, (2006). , 3230-3237.

[76] Cassell, O. C. S, et al. Vascularisation of tissue-engineered grafts: the regulation of angiogenesis in reconstructive surgery and in disease states. British Journal of Plastic Surgery, (2002). , 603-610.

[77] Unger, R. E, et al. Growth of human cells on a non-woven silk fibroin net: a potential for use in tissue engineering. Biomaterials, (2004). , 1069-1075.

[78] Kaigler, D, et al. VEGF Scaffolds Enhance Angiogenesis and Bone Regeneration in Irradiated Osseous Defects. Journal of Bone and Mineral Research, (2006). , 735-744.

[79] Murphy, W. L, et al. Bone regeneration via a mineral substrate and induced angiogenesis. Journal of Dental Research, (2004). , 204-210.

[80] Narayan, D, \& Venkatraman, S. S. Effect of pore size and interpore distance on endothelial cell growth on polymers. Journal of Biomedical Materials Research Part A, (2008). $\mathrm{A}(3)$ : , 710-718.

[81] Karageorgiou, V, \& Kaplan, D. Porosity of 3D biornaterial scaffolds and osteogenesis. Biomaterials, (2005). , 5474-5491. 
[82] Kim, S, \& Von Recum, H. Endothelial stem cells and precursors for tissue engineering: cell source, differentiation, selection, and application. Tissue engineering. Part B, Reviews, (2008). , 133-147.

[83] Melero-martin, J. M, et al. Engineering robust and functional vascular networks in vivo with human adult and cord blood-derived progenitor cells. Circulation Research, (2008). , 194-202.

[84] Urbich, C, \& Dimmeler, S. Endothelial progenitor cells- Characterization and role in vascular biology. Circulation Research, (2004). , 343-353.

[85] Usami, K, et al. Composite implantation of mesenchymal stem cells with endothelial progenitor cells enhances tissue-engineered bone formation. Journal of Biomedical Materials Research Part A, (2009). A(3): , 730-741.

[86] Seebach, C, et al. Endothelial Progenitor Cells and Mesenchymal Stem Cells Seeded onto beta-TCP Granules Enhance Early Vascularization and Bone Healing in a Critical-Sized Bone Defect in Rats. Tissue Engineering Part A, (2010). , 1961-1970.

[87] Liu, Y, et al. Contrasting Effects of Vasculogenic Induction Upon Biaxial Bioreactor Stimulation of Mesenchymal Stem Cells and Endothelial Progenitor Cells Cocultures in 3D Scaffolds Under In Vitro and In Vivo Paradigms for Vascularized Bone Tissue Engineering. Tissue Eng Part A, (2012). In Press.

[88] Liu, Y, et al. Vasculogenic and Osteogenesis-Enhancing Potential of Human Umbilical Cord Blood Endothelial Colony-Forming Cells. Stem Cells, (2012). , 1911-1924.

[89] Fuchs, S, et al. Contribution of outgrowth endothelial cells from human peripheral blood on in vivo vascularization of bone tissue engineered constructs based on starch polycaprolactone scaffolds. Biomaterials, (2009). , 526-534.

[90] Brown, B. N, et al. Macrophage polarization: an opportunity for improved outcomes in biomaterials and regenerative medicine. Biomaterials, (2012). , 3792-3802.

[91] Sica, A, \& Mantovani, A. Macrophage plasticity and polarization: in vivo veritas. J Clin Invest, (2012). , 787-795. 